\title{
FORMS OF HIGHER DEGREE PERMITTING COMPOSITION
}

\author{
S. PUMPLÜN
}

\begin{abstract}
Nondegenerate forms $N$ of degree $d$ on a unital nonassociative algebra $A$ over a ring $R$ which permit composition, i.e., satisfy $N(1)=1$ and $N(x y)=N(x) N(y)$ for all $x, y$ in $A$, are studied. These forms were first classified by Schafer over fields of characteristic 0 or $>d$. We investigate cubic and quartic nondegenerate forms which permit composition over certain rings and curves. Classes of highly degenerate cubic forms $N$ over fields which permit composition are constructed.
\end{abstract}

\section{InTRODUCTION}

Finite-dimensional forms of degree $d$ permitting composition which are defined over fields of characteristic 0 or $>d$ were classified by Schafer (see [S1] for an overview, [S2] for cubic forms and [S3] for forms of higher degree). Both the restriction on the dimension of the underlying vector space to be finite, and on the characteristic of the base field which were needed in Schafer's proofs, were later omitted, respectively relaxed, by McCrimmon [M1]. Forms permitting composition over arbitrary base rings instead of fields were first studied by Baumgartner and Bergmann [B-B] in 1974, McCrimmon [M2] in 1985 and Petersson $[\mathrm{P}]$ in 1993. McCrimmon studied quadratic forms permitting composition in the context of nonassociative algebras with a scalar involution. Petersson generalized the classical CayleyDickson doubling process, and completely classified composition algebras over arbitrary rings which, as modules, are finitely generated projective with full support. Baumgartner and Bergmann investigated nondegenerate cubic forms over arbitrary commutative unital rings satisfying a certain multiplicativity condition which is a canonical generalization of what we call "permitting composition". In particular, any unital nonassociative algebra over $R$ carrying a nondegenerate multiplicative cubic form mapping the unit element of the algebra onto the unit of the base ring, was shown to be alternative and algebraic [B-B, Satz 3 , Satz 4]. This result was extended to arbitrary multiplicative forms of higher degree over rings $R$ with $[d / 2] ! \in R^{\times}$by Legrand-Legrand [L-L]. Since there are no restrictions on the characteristic of the base ring, the more general concept of a form of degree $d$ as it was developed by Bergmann [B] was used (see also Roby [R]; the concept of forms developed by him comprises the one of Bergmann, both coincide for finitely generated projective modules). In the setting we consider, their forms are identical with ours and some of Schafer's results carry over verbatim to the setting of forms permitting composition over rings.

2000 Mathematics Subject Classification. Primary: 11E76, Secondary: 17A75, 17D05.

Key words and phrases. Forms of higher degree, alternative algebras, forms permitting composition, norm forms. 
Petersson was the first to investigate composition algebras over locally ringed spaces, thus initiating the study of nonassociative algebras over algebraic varieties. In particular, he generalized the classical Cayley-Dickson doubling process due to Albert $[\mathrm{A}]$ to this more general setting $[\mathrm{P}, 2.5]$. Composition algebras (defined on locally free modules of constant finite rank) were classified over curves of genus zero in $[\mathrm{P}, 4.4]$.

We study forms of degree $d \geq 3$ permitting composition which are defined over rings with $d ! \in R^{\times}$instead of fields. We also investigate unital nonassociative $\mathcal{O}_{X}$-algebras $\mathcal{A}$ over locally ringed spaces $X, d ! \in H^{0}\left(X, \mathcal{O}_{X}^{\times}\right)$, which carry a nondegenerate form $N: \mathcal{A} \rightarrow \mathcal{O}_{X}$ of degree $d \geq 3$ permitting composition, i.e., $N(x y)=N(x) N(y)$ for all sections $x, y$ of $\mathcal{A}$ over the same open subset of $X$ and $N\left(1_{\mathcal{A}}\right)=1$.

The contents of this paper are as follows. Let $R$ be a commutative ring such that $d ! \in R^{\times}$. Let $A$ be a unital nonassociative $R$-algebra which is finitely generated projective and faithful as an $R$-module. Suppose that $A$ carries a nondegenerate form $N$ of degree $d$ permitting composition. Notation and basic facts are given in Section 1. After some straightforward generalizations of results in [S3] to forms over $R$ in Section 2 , the cases $d=3$ and $d=4$ are considered separately in Section 4. In particular, we investigate the properties of $A$ over a domain. Examples of cubic and quartic forms over the rings $k[t]$ and $k\left[t, \frac{1}{t}\right]$ which permit composition are given in Section 5. In Section 6 our previous results are generalized to the setting of locally ringed spaces. In Section 7, algebras over a curve of genus zero which admit a nondegenerate cubic form $N$ permitting composition are listed. As an application, which shows how useful the theory of alternative algebras over varieties can be, we construct classes of unital alternative algebras of degree 3 over a field of characteristic not 2 or 3 with a highly degenerate form $N$ which permits composition in Section 8.

In the following, the standard terminology from algebraic geometry, see Hartshorne's book $[\mathrm{H}]$, is used.

\section{Preliminaries}

2.1. Let $R$ be a unital commutative associative ring. The rank of a finitely generated projective $R$-module $M$ is defined as $\sup \left\{\operatorname{rank}_{R_{P}} M_{P} \mid P \in \operatorname{Spec} R\right\}$. Let $A$ be a nonassociative unital $R$-algebra. The nucleus $\operatorname{Nuc}(A)=\{x \in A \mid[x, A, A]=[A, x, A]=[A, A, x]=0\}$ of $A$ is the set of those elements in $A$, which associate with all elements in $A$. The center of $A$ is the set of all elements which commute and associate with all elements of $A$; that is, the set $Z(A)=\{x \in \operatorname{Nuc}(A) \mid x y=y x$ for all $y \in A\}[$ S4, p. 14].

Remark 1. (i) An $R$-module $M$ has full support if $\operatorname{Supp}(M)=\left\{P \in \operatorname{Spec} R \mid M_{P} \neq 0\right\}=$ Spec $R$. Every nonassociative unital algebra over $R$ which is finitely generated projective with full support is faithful; i.e., $r A=0$ implies $r=0$.

(ii) If $A$ is finitely generated projective and faithful as $R$-module, $R 1_{A}$ is a direct summand of $A$ : The map $\epsilon: R \rightarrow A, \epsilon(r)=r 1_{A}$ has a section if and only if $\operatorname{Hom}\left(\epsilon, 1_{A}\right)$ is surjective. Since $A_{P}$ has positive dimension, the map is surjective modulo any maximal ideal, which implies the assertion (see the proof of [Kn1, I.(1.3.5)]).

Unless stated otherwise, the term " $R$-algebra" refers to unital nonassociative algebras which are finitely generated projective and faithful as $R$-modules. 
2.2. An $R$-algebra $C$ is called a composition algebra if it admits a quadratic form $N: C \rightarrow R$ such that $N\left(1_{C}\right)=1$ which permits composition and whose induced symmetric bilinear form is nondegenerate; i.e., it determines an $R$-module isomorphism $C \stackrel{\sim}{\longrightarrow} \check{C}=\operatorname{Hom}_{R}(C, R) . C$ is alternative and quadratic. The quadratic form $N$ is uniquely determined by $C$ and called the norm of $C$. Composition algebras over $R$ only exist in ranks 1, 2, 4 or 8 . Those of constant rank 2 are called quadratic étale $R$-algebras. Those of constant rank 4 are called quaternion algebras and the ones of constant rank 8 are called octonion algebras. If there is a nondegenerate quadratic form $N: A \rightarrow R$ on a unital nonassociative $R$-algebra $A$ permitting composition, then $A$ is a composition algebra over $R$ and of rank 1, 2, 4 or $8[\mathrm{P}]$.

2.3. Let $d$ be a positive integer and assume $d ! \in R^{\times}$. Let $M$ be a finitely generated projective $R$-module. A form of degree $d$ (on $M$ ) over $R$ is a map $N: M \rightarrow R$ such that $N(a x)=a^{d} N(x)$ for all $a \in R, x \in M$, and where the map $\theta: M \times \cdots \times M \rightarrow R$ defined by

$$
\theta\left(x_{1}, \ldots, x_{d}\right)=\frac{1}{d !} \sum_{1 \leq i_{1}<\cdots<i_{l} \leq d}(-1)^{d-l} N\left(x_{i_{1}}+\cdots+x_{i_{l}}\right)
$$

is a $d$-linear form over $R$ (the range of summation of $l$ being $1 \leq l \leq d$ ). $\theta$ is called the symmetric d-linear form associated with $N$ and $(M, \theta)$ a $d$-linear space. Obviously, $N(x)=\theta(x, \ldots, x)$. Note that a symmetric $R$-module homomorphism $M \otimes \cdots \otimes M \rightarrow R$ or an $R$-module homomorphism $S_{d}(M) \rightarrow R$, where $S_{d}(M)$ is the symmetric algebra of $M$, also define a symmetric $d$-linear form.

$N$ (or, respectively, the associated $d$-linear form $\theta$ ) is called nondegenerate, if the map $M \rightarrow \operatorname{Hom}_{R}(M \otimes \cdots \otimes M, R)((d-1)$-copies of $M)$

$$
\theta_{x_{1}}\left(x_{2} \otimes \cdots \otimes x_{d}\right)=\theta\left(x_{1}, x_{2}, \ldots, x_{d}\right)
$$

is injective (cf. Keet [K, p. 4946]). This concept of nondegeneracy is not invariant under base change. A stronger notion, invariant under base change, is to require that $\theta_{x_{1}} \otimes k(P)$ is injective for all $P \in \operatorname{Spec} R$. This is equivalent to saying that $\theta_{x_{1}}$ is an isomorphism of $M$ onto a direct sumand of $\operatorname{Hom}_{R}\left(M^{\otimes(d-1)}, R\right)$. Both notions, however, are equivalent for forms permitting composition, see Lemma 1 (i).

Two $d$-linear spaces $\left(M_{i}, \theta_{i}\right), i=1,2$ are called isomorphic (written $\left(M_{1}, \theta_{1}\right) \cong\left(M_{2}, \theta_{2}\right)$ or just $\left.\theta_{1} \cong \theta_{2}\right)$ if there exists an $R$-module isomorphism $f: M_{1} \rightarrow M_{2}$ such that $\theta_{2}\left(f\left(x_{1}\right), \ldots, f\left(x_{d}\right)\right)=\theta_{1}\left(x_{1}, \ldots, x_{d}\right)$ for all $x_{1}, \ldots, x_{d} \in M_{1}$.

The orthogonal sum $\left(M_{1}, \theta_{1}\right) \perp\left(M_{2}, \theta_{2}\right)$ of $\left(M_{i}, \theta_{i}\right), i=1,2$, is defined to be the $R$-module $M_{1} \oplus M_{2}$ together with the $d$-linear form $\left(\theta_{1} \perp \theta_{2}\right)\left(u_{1}+x_{1}, \ldots, u_{d}+x_{d}\right)=$ $\theta_{1}\left(u_{1}, \ldots, u_{d}\right)+\theta_{2}\left(x_{1}, \ldots, x_{d}\right)$. A $d$-linear space $(M, \theta)$ is called decomposable, if $(M, \theta) \cong$ $\left(M_{1}, \theta_{1}\right) \perp\left(M_{2}, \theta_{2}\right)$ for two non-zero $d$-linear spaces $\left(M_{i}, \theta_{i}\right), i=1,2$. A non-zero $d$-linear space $(M, \theta)$ is called indecomposable if it is not decomposable. We distinguish between indecomposable ones and absolutely indecomposable ones; i.e., $d$-linear spaces which stay indecomposable under base change.

2.4. Let $X$ be a locally ringed space with structure sheaf $\mathcal{O}_{X}$. For $P \in X$ let $\mathcal{O}_{P, X}$ be the local ring of $\mathcal{O}_{X}$ at $P$ and $m_{P}$ the maximal ideal of $\mathcal{O}_{P, X}$. The corresponding residue class field is denoted by $k(P)=\mathcal{O}_{P, X} / m_{P}$. For an $\mathcal{O}_{X}$-module $\mathcal{F}$ the stalk of $\mathcal{F}$ at $P$ 
is denoted by $\mathcal{F}_{P} . \mathcal{F}$ is said to have full support if $\operatorname{Supp} \mathcal{F}=X$; i.e., if $\mathcal{F}_{P} \neq 0$ for all $P \in X$. We call $\mathcal{F}$ locally free of finite rank if for each $P \in X$ there is an open neighborhood $U \subset X$ of $P$ such that $\left.\mathcal{F}\right|_{U}=\mathcal{O}_{U}^{r}$ for some integer $r \geq 0$. The rank of $\mathcal{F}$ is defined to be

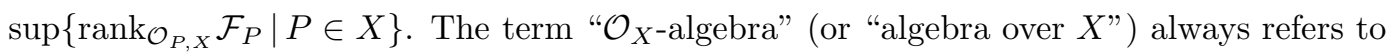
nonassociative $\mathcal{O}_{X}$-algebras which are unital and locally free of finite rank as $\mathcal{O}_{X}$-modules.

2.5. Let $\mathcal{C}$ be an $\mathcal{O}_{X}$-algebra. $\mathcal{C}$ is called a composition algebra over $X$ if it has full support and if there exists a nondegenerate quadratic form $N: \mathcal{C} \rightarrow \mathcal{O}_{X}$ (i.e., the induced symmetric bilinear form $N(u, v)=N(u+v)-N(u)-N(v)$ determines a module isomorphism $\mathcal{C} \stackrel{\sim}{\longrightarrow}$ $\left.\check{\mathcal{C}}=\mathcal{H o m}\left(\mathcal{C}, \mathcal{O}_{X}\right)\right)$, such that $N(u v)=N(u) N(v)$ for all sections $u, v$ of $\mathcal{C}$ over the same open subset of $X[\mathrm{P}, 1.6]$.

The form $N$ is uniquely determined by these conditions and called the norm of $\mathcal{C}$. It is denoted by $N_{\mathcal{C}}$. Given an algebra $\mathcal{C}$ over $X$ and a quadratic form $N: \mathcal{C} \rightarrow \mathcal{O}_{X}$, the algebra $\mathcal{C}$ is a composition algebra over $X$ with norm $N$ if and only if $\mathcal{C}_{P}$ is a composition algebra over $\mathcal{O}_{P, X}$ with norm $N_{P}$ for all $P \in X$. Composition algebras over $X$ are invariant under base change, and exist only in ranks 1, 2, 4 or 8 . A composition algebra of constant rank 2 (resp. 4 or 8 ) is called a quadratic étale algebra (resp. quaternion algebra or an octonion algebra). A composition algebra over $X$ of constant rank is called split, if it contains a composition subalgebra isomorphic to $\mathcal{O}_{X} \oplus \mathcal{O}_{X}[\mathrm{P}, 1.7,1.8]$.

If $X$ is an $R$-scheme with structure morphism $\tau: X \rightarrow \operatorname{Spec} R$, then a composition algebra $\mathcal{C}$ over $X$ is defined over $R$ if there exists a composition algebra $C$ over $R$ such that $\mathcal{C}=$ $\tau^{*} C \cong C \otimes \mathcal{O}_{X}$. There exists a generalized Cayley-Dickson doubling $\operatorname{Cay}(\mathcal{D}, \mathcal{P}, N)$ for a composition algebra $\mathcal{D}$ of constant rank $\leq 4$ over a locally ringed space $[\mathrm{P}, 2.3,2.4,2.5]$.

2.6. Let $d ! \in H^{0}\left(X, \mathcal{O}_{X}^{\times}\right)$. Let $\mathcal{M}$ be an $\mathcal{O}_{X}$-module which is locally free of finite rank. A form of degree $d$ (on $\mathcal{M}$ ) over $\mathcal{O}_{X}$ is a map $N: \mathcal{M} \rightarrow \mathcal{O}_{X}$ such that $N(a x)=a^{d} N(x)$ for all sections $a$ of $\mathcal{O}_{X}, x$ of $\mathcal{M}$ over the same open subset of $X$, and where the map $\theta: \mathcal{M} \times \cdots \times \mathcal{M} \rightarrow \mathcal{O}_{X}$ defined by

$$
\theta\left(x_{1}, \ldots, x_{d}\right)=\frac{1}{d !} \sum_{1 \leq i_{1}<\cdots<i_{l} \leq d}(-1)^{d-l} N\left(x_{i_{1}}+\cdots+x_{i_{l}}\right)
$$

$(1 \leq l \leq d)$ is a $d$-linear form over $\mathcal{O}_{X} \cdot \theta$ is called the symmetric d-linear form associated with $N$. (This definition of associated $d$-linear form deviates from the one used for the associated symmetric bilinear form in 2.5 or $[\mathrm{P}]$ by the factor $1 / 2$ which was omitted in order to be able to include the case that $2 \notin H^{0}\left(X, \mathcal{O}_{X}^{\times}\right)$in the classification result for composition algebras). A form $N$ of degree $d \geq 3$ (or, respectively, its associated $d$-linear form $\theta)$ is called nondegenerate, if the map $\mathcal{M} \rightarrow \mathcal{H o m}_{X}\left(\mathcal{M} \otimes \cdots \otimes \mathcal{M}, \mathcal{O}_{X}\right)((d-1)$-copies of $\mathcal{M})$,

$$
\theta_{x_{1}}\left(x_{2} \otimes \cdots \otimes x_{d}\right)=\theta\left(x_{1}, x_{2}, \ldots, x_{d}\right)
$$

is injective.

Two $d$-linear spaces $\left(\mathcal{M}_{i}, \theta_{i}\right)(i=1,2)$ are called isomorphic if there exists an $\mathcal{O}_{X}$-module isomorphism $f: \mathcal{M}_{1} \rightarrow \mathcal{M}_{2}$ such that $\theta_{2}\left(f\left(v_{1}\right), \ldots, f\left(v_{d}\right)\right)=\theta_{1}\left(v_{1}, \ldots, v_{d}\right)$ for all sections $v_{1}, \ldots, v_{d}$ of $\mathcal{M}_{1}$ over the same open subset of $X$. 
The orthogonal sum $\left(\mathcal{M}_{1}, \theta_{1}\right) \perp\left(\mathcal{M}_{1}, \theta_{2}\right)$ of $\left(\mathcal{M}_{i}, \theta_{i}\right), i=1,2$, is defined to be the $\mathcal{O}_{X}$-module $\mathcal{M}_{1} \oplus \mathcal{M}_{2}$ together with the $d$-linear form $\left(\theta_{1} \perp \theta_{2}\right)\left(u_{1}+v_{1}, \ldots, u_{d}+v_{d}\right)=$ $\theta_{1}\left(u_{1}, \ldots, u_{d}\right)+\theta_{2}\left(v_{1}, \ldots, v_{d}\right)$. A $d$-linear space $(\mathcal{M}, \theta)$ is called decomposable, if $(\mathcal{M}, \theta) \cong$ $\left(\mathcal{M}_{1}, \theta_{1}\right) \perp\left(\mathcal{M}_{2}, \theta_{2}\right)$ for two non-zero $d$-linear spaces $\left(\mathcal{M}_{i}, \theta_{i}\right), i=1,2$. A non-zero $d$-linear space $(\mathcal{M}, \theta)$ is called indecomposable if it is not decomposable. We will distinguish between indecomposable ones and absolutely indecomposable ones; i.e., $d$-linear spaces which stay indecomposable under base change.

\section{Forms PERMitTing COMPOSITION}

Large parts of the results and proofs of [S3] can be generalized verbatim to the case where the base field is replaced by an arbitrary commutative ring $R$ with $d ! \in R^{\times}$. We briefly summarize them for the sake of the reader.

Let $R$ be a ring with $d ! \in R^{\times}$. Unless explicitly stated otherwise, the term " $R$-algebra" refers to unital nonassociative algebras which are finitely generated projective and faithful as $R$-modules. Let $A$ be an algebra over $R, 1=1_{A}$ the unit element of $A$ and $N: A \rightarrow R$ a form of degree $d$ permitting composition; i.e.,

$$
\theta(x y, \ldots, x y)=\theta(x, \ldots, x) \theta(y, \ldots, y)
$$

for all $x, y \in A$ and $N\left(1_{A}\right)=1$. Linearizing this equation in $x$ and then in $y$ we obtain

$$
\theta\left(x_{1} y, \ldots, x_{d} y\right)=\theta\left(x_{1}, \ldots, x_{d}\right) N(y),
$$

and

$$
\sum_{\sigma} \theta\left(x_{1} y_{\sigma(1)}, \ldots, x_{d} y_{\sigma(d)}\right)=d ! \theta\left(x_{1}, \ldots, x_{d}\right) \theta\left(y_{1}, \ldots, y_{d}\right)
$$

where $\sigma$ ranges over all the permutations in $S_{d}$. This implies

$$
\theta\left(x y_{1}, \ldots, x y_{d}\right)=N(x) \theta\left(y_{1}, \ldots, y_{d}\right)
$$

by symmetry $[\mathrm{S} 3,(5),(6)$ and $(7)]$. (Indeed, this observation does not require $A$ to have a unit element.) For $i=1, \ldots, d$ we define a form $T_{i}: A \rightarrow R$ of degree $i$ via

$$
T_{i}(x)=\left(\begin{array}{c}
d \\
i
\end{array}\right) \theta(x, \ldots, x, 1, \ldots, 1) \quad(i \text {-times } x)
$$

Then

$$
N(x)=T_{d}(x) \text { and } T_{1}(x)=d \theta(x, 1, \ldots, 1) .
$$

Put $T_{0}(x)=1$ and $T_{d+q}(x)=0$ for $q>0$. The form $T: A \rightarrow R, T(x)=T_{1}(x)$ is called the trace. Define $A_{0}=\operatorname{ker} T$. $A$ can be written as the direct sum of $R$-modules

$$
A=R 1_{A} \oplus A_{0} .
$$

From now let $d \geq 3$, unless explicitly stated otherwise.

A $d$-linear form $\theta$ is invariant under all left and right multiplications with elements of trace zero, if

$$
\theta\left(x_{1} a, \ldots, x_{d}\right)+\theta\left(x_{1}, x_{2} a, \ldots, x_{d}\right)+\cdots+\theta\left(x_{1}, \ldots, x_{d} a\right)=0
$$

and

$$
\theta\left(a x_{1}, \ldots, x_{d}\right)+\theta\left(x_{1}, a x_{2}, \ldots, x_{d}\right)+\cdots+\theta\left(x_{1}, \ldots, a x_{d}\right)=0
$$


for all $x_{i} \in A$ and for all elements $a \in A$ of trace zero.

$B(x, y)=T(x y)$ is a symmetric bilinear form on $A$ which is associative, and if $N$ is nondegenerate, then so is $B$. $A$ is alternative; i.e., $x^{2} y=x(x y)$ and $y x^{2}=(y x) x$ for all elements $x, y \in A$. Every element $x \in A$ satisfies

$$
x^{d}-T_{1}(x) x^{d-1}+T_{2}(x) x^{d-2}-\cdots+(-1)^{d} T_{d}(x) 1=0
$$

([S3, Theorem 2, Theorem 3], for $d=2$ this was shown in [M2, 4.6]).

Remark 2. (i) If $N$ is degenerate, the algebra $A$ need not be alternative; for a counterexample for $d=2$ see [M2, 4.13].

(ii) Baumgartner and Bergmann studied cubic forms $N$ on unital nonassociative algebras $A$ over arbitrary rings which they called multiplicative, and called such a pair $(A, N)$ a composition algebra of third degree. Each nondegenerate composition algebra (in the sense of [B-B]) of degree 3 over an arbitrary ring is alternative [B-B, Satz 3]. This result for composition algebras of third degree in the sense of [B-B] was later generalized by Legrand-Legrand [L-L] to multiplicative forms of higher degree over rings with $[d / 2]$ ! invertible. Their definition of forms is different from ours in order to accommodate the case that $d$ ! is not invertible in $R$. Over fields of characteristic not 2 or 3 , or over the rings considered here, the different concepts of forms coincide and a form $N$ permitting composition which satisfies $N\left(1_{A}\right)=1$ corresponds to a multiplicative form. For the above equation in a more general context, see [L-L, Corollaire 2.2].

Theorem 1. Suppose that $R$ is a domain. Let $A$ be an $R$-algebra, $\theta: A \times \cdots \times A \rightarrow R$ a nondegenerate symmetric d-linear form on $A$ and let the trace $T: A \rightarrow R$ be defined as above. If $\theta$ is invariant under all left and right multiplications corresponding to elements of trace zero, then $A$ is semiprime; i.e., $I^{2} \neq 0$ for each non-zero ideal I in $A$.

For $d>2$ the proof can be found in the proof of [S3, Theorem 1 (c)]. A similar argument holds for $d=2$.

Corollary 1. Suppose that $R$ is a domain and $A$ an $R$-algebra with be a nondegenerate form $N: A \rightarrow R$ of degree $d \geq 2$ permitting composition. Then $A$ is a semiprime alternative algebra over $R$. If $I$ is a minimal ideal of $A$ and $I^{2} \neq(0)$, then $I$ is simple and $I^{2}=I$. Moreover, $I$ is either a simple associative ring or an octonion algebra over its center. In the latter case, $A=I \oplus I^{\prime}$ holds as an ideal direct sum.

Proof. The first assertion follows immediately from Theorem 1 using that the $d$-linear form associated with $N$ is invariant under all left and right multiplications corresponding to elements of trace zero, the second from [S12, Theorem A, Lemma 2.1, Theorem B].

Remark 3. Suppose $d \geq 2$ and that the ring $A$ satisfies the descending chain condition on right ideals. By Corollary $1, A$ is a semiprime alternative algebra over $R$. By [Sl1, Theorem $\mathrm{B}], A$ is expressible, unique up to order, as an ideal direct sum $A=A_{1} \oplus \cdots \oplus A_{r}$ of minimal ideals $A_{i}$ where each $A_{i}$ is either an octonion algebra over its center or a simple artinian associative ring. If every non-zero ideal of $A$ contains a minimal ideal of $A$ and $A$ is purely alternative (i.e., has no non-zero nuclear ideals), then $A$ is expressible, unique up to order, 
as an ideal direct sum $A=A_{1} \oplus \cdots \oplus A_{r}$ where each $A_{i}$ is an octonion algebra over its center [Sl2, Theorem A].

Lemma 1. (i) $N(P)$ is nondegenerate for all $P \in \operatorname{Spec} R$.

(ii) If $A$ is an associative algebra then $A$ is separable. If $A$ is also central then it is an Azumaya algebra over $R$.

Proof. (i) Since $N$ is nondegenerate, so is $T$ and thus also $T(P)$ for all $P \in X$. The fact that $T(P)$ is nondegenerate is equivalent to $N(P)$ being nondegenerate ([B, Satz 3] or [B-B, p. 327] for cubic forms) implying that $N(P)$ is a nondegenerate form for all $P \in X$.

(ii) $A$ is finitely generated as $R$-module by assumption, thus $A$ is separable over $R$ if and only if $A / m A$ is a separable $R / m$-algebra for all $m \in \operatorname{Max} R$ [Kn1, III. (5.1.10)]. This holds, since $A(P)$ admits a nondegenerate form of degree $d$ permitting composition for all $P \in \operatorname{Spec} R$. Thus $A(P)$ is a finite dimensional separable $k(P)$-algebra for all $P \in \operatorname{Spec} R$ by [S1, Theorem 3]. Hence $A$ is a separable associative $R$-algebra, finitely generated as an $R$-module. If $A$ is central, then $A$ is central, separable and finitely generated faithfully projective as an $R$-module, therefore an Azumaya algebra by [Kn1, III. (5.1.1)].

Thus, if $N$ permits composition, by (i) the two different notions of nondegeneracy introduced in 2.3 are equivalent.

Remark 4. Assume $A$ has center $R^{\prime}$ larger than $R$ in the situation of Lemma 1 (ii). Then $R^{\prime}$ is a separable ring extension of $R$. View $A$ as an $R^{\prime}$-algebra. $A$ is finitely generated and faithful as an $R^{\prime}$-module. In case $A$ is projective and separable also as an $R^{\prime}$-module, $A$ is an Azumaya algebra over $R^{\prime}$ [Kn1, III, (5.1.1)].

Proposition 1. $A(P)$ is a finite-dimensional separable algebra over $k(P)$ and

(i) if $N$ is a cubic form, then $A$ has rank 1,2, 3, 5 or 9 over $R$;

(ii) if $N$ is a quartic form, then $A$ has rank 1, 2, 3, 4, 5, 6, 8, 9, 10, 12 or 16 over $R$.

Proof. For all $P \in \operatorname{Spec} R$ the residue class algebra $A(P)$ is a non-zero alternative algebra over the residue class field $k(P)$ together with a nondegenerate form $N(P): A(P) \rightarrow k(P)$ which permits composition. Therefore $A(P)$ is a finite-dimensional separable algebra over $k(P)$ [S1, Theorem 3]. Hence, if $N$ is a cubic form, then $A$ must have rank 1, 2, 3, 5 or 9 , and if it is a quartic form then $A$ must have rank 1, 2, 3, 4, 5, 6, 8, 9, 10, 12 or 16 [S2, S3].

Corresponding statements can be derived for forms of higher degree than 4 .

3.1. For this section we assume that $R$ is a domain.

Lemma 2. Suppose that $A$ contains an idempotent $e \neq 0,1$. Then 1 and $e$ are linearly independent over $R$.

Proof. Let $1 a+e b=0$ for $a, b \in R$. Multiplication with $e$ implies $(a+b) e=0$ and hence $a+b=0$, since $A$ is a projective $R$-module, thus torsion free as an $R$-module. Now $b=-a$, so $0=1 a+e b=(1-e) a$ yields $a=0$ since $1-e \neq 0$, hence also $b=0$. 
Suppose that $A$ contains an idempotent $e \neq 0,1$. Then

$$
x^{d}-T_{1}(x) x^{d-1}+T_{2}(x) x^{d-2}-\cdots+(-1)^{d} T_{d}(x) 1=0
$$

for all $x \in A$ implies that

$$
1-T(e)+T_{2}(e)-\cdots+(-1)^{d-1} T_{d-1}(e) 1=0,
$$

and $N(e)=0$, since 1 and $e$ are linearly independent. As in [S3, p. 785] we obtain the equations

$$
(j+1) T_{j+1}(e)=(T(e)-j) T_{j}(e)
$$

for $j=1, \ldots, d-1[\mathrm{~S} 3,(38)]$, and

$$
T_{i}(e)=\left(\begin{array}{c}
m \\
i
\end{array}\right)
$$

for $i=1, \ldots, m[\mathrm{~S} 3,(40)]$, where $m$ is the least integer such that $T_{m+1}(e)=\cdots=T_{d}(e)=0$.

These are needed for the proof of the next result, which closely follows the one given in [S3] for Theorem 4.

Theorem 2. If

$$
A=A_{1} \oplus \cdots \oplus A_{r}
$$

is the direct sum of ideals $A_{i} \neq 0$ in $A$, then

$$
N(x)=N_{1}\left(x_{1}\right) \cdots N_{2}\left(x_{r}\right)
$$

where $x=x_{1}+\cdots+x_{r}, x_{i} \in A_{i}$. Each $N_{i}$ is a nondegenerate form of degree $d_{i}$ on $A_{i}$ which permits composition and $d=d_{1}+\cdots+d_{r}$. If $r \geq 2$ then $N$ is absolutely indecomposable.

Proof. Assume that $A=G \oplus G^{\prime}$ with $G \neq 0, G^{\prime} \neq 0$ ideals. Write $1=e+e^{\prime}$ with $e \in G$ and $e^{\prime} \in G^{\prime}$. Then $e \neq 0,1$ (resp. $e^{\prime} \neq 0,1$ ) is the unit element of $G$ (resp. of $G^{\prime}$ ) and as such it is an idempotent in $A$. For $g \in G, g^{\prime} \in G^{\prime}$ define

$$
N_{G}(g)=N(g+e), N_{G^{\prime}}\left(g^{\prime}\right)=N\left(e+g^{\prime}\right) .
$$

For any $x=g+g^{\prime} \in A$ we get

$$
N(x)=N_{G}(g) N_{G^{\prime}}\left(g^{\prime}\right)
$$

and

$$
N_{G}\left(g_{1} g_{2}\right)=N_{G}\left(g_{1}\right) N_{G}\left(g_{2}\right)
$$

for all $g_{1}, g_{2} \in G$. By showing that

$$
N_{G}(g)=T_{m}(g) \text { for all } g \in G, \text { where } m=T(e),
$$

$N_{G}$ is proved to be a form of degree $m$ over $G$. (The fact that 1 and $e$ are linearly independent is needed to obtain $T(e)=m$.) Symmetrically, the same formulas hold for $N_{G^{\prime}}$, so that $N(x)$ is a product of forms of degree $m$ and $m^{\prime}$ permitting composition and $d=m+m^{\prime}$. The proof that $N_{G}(g)=T_{m}(g)$ for all $g \in G$, with $m=T(e)$ is the same as given in [S3]. Both $N_{G}$ and $N_{G^{\prime}}$ are nondegenerate, see the proof in [S3, p. 787, 788].

This argument can be repeated finitely often and since $A=A_{1} \oplus \cdots \oplus A_{r}$ with $A_{i} \neq 0$ ideals, we obtain the assertion by induction.

That $N$ is absolutely indecomposable follows from [Pr, 5.1]. 
If $R$ is a field, it is well-known that any form on a simple alternative algebra which permits composition is a power of the generic norm of the algebra. If $R$ is a ring, it can happen that an algebra admits more than one nondegenerate form permitting composition, see [M2].

Remark 5. (i) Let $A=A_{1} \oplus A_{2}$ be a direct sum of two ideals. If $A$ is finitely generated projective as an $R$-module then so are $A_{1}$ and $A_{2}$.

(ii) If $d=3$ (resp. 4) in Theorem 2 , then $A$ is the direct sum of at most three (resp. four) non-zero ideals which all are unital nonassociative algebras admitting a form of degree 1 or 2 (resp. 1, 2 or 3 ) permitting composition.

\section{Cubic And Quartic Forms}

A nondegenerate cubic (or quartic) form $N: A \rightarrow R$ permitting composition can only exist in rank 1, 2, 3, 5 or 9 (or in rank 1, 2, 3, 4, 5, 6, 8, 9, 10, 12 or 16) by Proposition 1. [S3, Lemma 1, 2, 3] applied to the residue class algebras imply the next two lemmas:

Lemma 3. Suppose that there exists a cubic form $N: A \rightarrow R$ permitting composition.

(i) If $A$ is a non-split quadratic étale algebra over $R$, then the residue class algebras $A(P)$ must be split for all $P \in \operatorname{Spec} R$.

(ii) A cannot be a quaternion or octonion algebra over $R$.

Lemma 4. Let $A$ be a composition algebra over $R$ of constant rank with norm $n_{A}$. Suppose that there exists a quartic form $N: A \rightarrow R$ permitting composition.

(i) If $A$ is a quadratic étale algebra and $A(P)$ is a quadratic field extension for all $P \in \operatorname{Spec} R$, or if $A$ has rank $\geq 4$ then $N(P)(x)=n_{A(P)}(x)^{2}$ for all $P \in \operatorname{Spec} R$ and $x \in A(P)$.

(ii) A cannot be an Azumaya algebra over $R$ of rank 9 .

(iii) If $A$ is a cubic ring extension of $R$, then $A(P)$ is not a cubic field extension of $k(P)$. for all $P \in \operatorname{Spec} R$.

Theorem 3. (i) Let $A$ be an $R$-algebra of constant rank such that there exists a cubic form $N$ on A permitting composition. Suppose that for each $P \in \operatorname{Spec} R$ there exists an element $u \in A \otimes_{R} k(P)$ such that $1, u, u^{2}$ are linearly independent over $k(P)$ (by Theorem 2, $A$ is alternative, so the powers of $A$ are unambiguous). Let $M, Q$ and $L$ be a cubic, a quadratic and a linear form from $A$ to $R$ satisfying

$$
x^{3}-L(x) x^{2}+Q(x) x-M(x) 1=0
$$

for all $x \in A$. Then $M=N, S=T_{2}$ and $L=T$.

(ii) Let $A$ be an R-algebra of constant rank such that there exists a quartic form $N$ on $A$ permitting composition. Suppose that for each $P \in \operatorname{Spec} R$ there exists an element $u \in$ $A \otimes_{R} k(P)$ such that $1, u, u^{2}, u^{3}$ are linearly independent over $k(P)$. Let $S, M, Q$ and $L$ be a quartic, cubic, quadratic and a linear form from $A$ to $R$ satisfying

$$
x^{4}-L(x) x^{3}+Q(x) x^{2}-M(x) x+S(x) 1=0
$$

for all $x \in A$. Then $S=N, M=T_{3}, Q=T_{2}$ and $L=T$. 
Part (i) is a Corollary of [Ach, 1.12] applied to the Jordan algebra $A^{+}$determined by $A$, (ii) can be proved analogously (if more tediously). Indeed, both results remain true even if we remove the restriction on $R$ to satisfy $d ! \in R^{\times}$and work with the more general notion of a form of higher degree as given in $[R]$.

Remark 6. Suppose that $A$ is an Azumaya algebra of constant rank 9 over $R$. For each $P \in \operatorname{Spec} R$ there exists an element $u \in A(P)$ such that $1, u, u^{2}$ are linearly independent over $k(P)$, since $A(P)$ is a central simple algebra of degree 3 . Since the reduced norm $n$ and trace $t$ of $A$ satisfy $x^{3}-t(x) x^{2}+q(x) x-n(x) 1=0$ with $q$ a quadratic form, it follows that $M=n($ and $L=t)$.

Suppose that $A$ is a cubic ring extension of $R$. For each $P \in \operatorname{Spec} R$ there exists an element $u \in A(P)$ such that $1, u, u^{2}$ are linearly independent over $k(P)$, since $A(P)$ is a cubic étale algebra over $k(P)$. Since the reduced norm $n$ and trace $t$ of $A$ satisfy $x^{3}-$ $t(x) x^{2}+q(x) x-n(x) 1=0$ with $q$ a quadratic form, it follows again that $M=n$ (and $L=t)$.

Analogous arguments show that also for a quartic separable ring extension of $R$ and for an Azumaya algebra of constant rank 16 over $R$, any quartic form on $A$ which permits composition must be uniquely determined and be the norm of the algebra, if $k(P)$ is an infinite field for all $P \in \operatorname{Spec} R$.

For the rest of this section, let $R$ be a domain.

Proposition 2. Let $N: A \rightarrow R$ be a cubic form on A permitting composition. Then $(A, N)$ is one of the following:

(i) $A=R$ and $N(x)=x^{3}$;

(ii) $A$ is a separable commutative associative $R$-algebra of rank 2 or 3 , and $N$ is absolutely indecomposable; if $A$ is a non-split quadratic étale algebra over $R$, then the residue class algebras $A(P)$ must be split for all $P \in \operatorname{Spec} R$, if $A$ has rank 3, then $A(P)$ is a cubic étale algebra over $k(P)$ for all $P \in \operatorname{Spec} R$.

(iii) A has rank 5 and is a separable associative, but not commutative, $R$-algebra and $N$ is absolutely indecomposable.

(iv) If $A$ has rank 9 and is associative, then it is an Azumaya algebra over $R$ and $N$ is its - uniquely determined - norm. If $A$ has rank 9 and is not associative, then $A$ is not commutative and $N$ is absolutely indecomposable.

Proof. Let $m$ denote the rank of $A$, then $m \in\{1,2,3,5,9\}$.

(1) If $m=1$, then $A=R$ and $N(x)=x^{3}$.

(2) If $m=2$, then $A(P) \cong k(P) \oplus k(P)$ is commutative, associative and $N(P)\left(x_{1}+\right.$ $\left.x_{2}\right)=x_{1} x_{2}^{2}$ is absolutely indecomposable for all $P \in \operatorname{Spec} R$. Thus all commutators and associators lie in $I A$ where $I$ is the nil radical of $R$. Since $R$ is a domain, $I=0$ and $A$ itself must be a commutative associative $R$-algebra. $N(P)\left(x_{1}+x_{2}\right)=$ $x_{1} x_{2}^{2}$ is absolutely indecomposable for all $P \in \operatorname{Spec} R$, hence $N$ is an absolutely indecomposable form. The rest follows from Lemma 3. 
(3) If $m=3$, then $A(P)$ is a cubic étale algebra over $k(P)$ and $N(P)$ is its (absolutely indecomposable) norm for all $P \in \operatorname{Spec} R$. Therefore $N$ is absolutely indecomposable and, by the same argument as above, $A$ must be commutative and associative.

(4) If $m=5$, then $A(P) \cong k(P) \oplus$ "some quaternion algebra over $k(P)$ " for all $P \in$ Spec $R$. Therefore $A(P)$ is associative and not commutative and $N(P)$ is absolutely indecomposable for all $P \in \operatorname{Spec} R$. Thus $A$ is associative by the same argument as above, and not commutative. $N$ is absolutely indecomposable.

(5) If $m=9$ we distinguish two cases: If $A$ is associative, then $A(P)$ is a central simple algebra of degree 3 over $k(P)$ for all $P \in \operatorname{Spec} R$ and $N(P)$ is its norm. Therefore $A$ is an Azumaya algebra over $R$ and $N$ is its - uniquely determined - norm.

If $A$ is not associative, then $A\left(P_{0}\right) \cong k\left(P_{0}\right) \oplus$ "some octonion algebra over $k\left(P_{0}\right)$ " for some $P_{0} \in \operatorname{Spec} R$ and $N\left(P_{0}\right)$ is absolutely indecomposable. Thus $A$ is not associative and not commutative and $N$ is again absolutely indecomposable.

Example 1. Over rings, the first Tits construction [P-R, Theorem 3.5] starting with $R$ can be generalized as follows [Ach, 2.25]: Let $L \in{ }_{3} \mathrm{Pic} R$ and $N_{L}: L \rightarrow R$ a nondegenerate cubic form, let $L^{\vee}=\operatorname{Hom}_{R}(L, R)$ be its dual and $\langle w, \check{w}\rangle=\check{w}(w)$ the canonical pairing $L \times \check{L} \rightarrow R$. There exists a uniquely determined cubic norm $\check{N}_{L}: L^{\vee} \rightarrow R$ and uniquely determined adjoints $\sharp: L \rightarrow L^{\vee}$ and $\sharp: L^{\vee} \rightarrow L$ such that

(1) $\left\langle w, w^{\sharp}\right\rangle=N_{L}(w) 1 ;$

(2) $\left\langle\check{w}^{\sharp}, \check{w}\right\rangle=\check{N}_{L}(\check{w}) 1$;

(3) $w^{\sharp \sharp}=N_{L}(w) w$;

for $w$ in $L, \check{w}$ in $L^{\vee}$. Moreover,

(1) $\check{w}^{\sharp \sharp}=\check{N}_{L}(\check{w}) \check{w}$;

(2) $\langle w, \check{w}\rangle^{2}=\left\langle\check{w}^{\sharp}, w^{\sharp}\right\rangle$;

(3) $\langle w, \check{w}\rangle^{3}=N_{L}(w) \check{N}_{L}(\check{w})$;

(4) $\langle w, \check{w}\rangle w=3\langle w, \check{w}\rangle w-w^{\sharp} \check{x} \check{w}$

for $w, w^{\prime}$ in $L, \check{w}$ in $L^{\vee}$ [Ach, 2.13]. Define

$$
\begin{aligned}
& \widetilde{\mathcal{J}}=R \oplus L \oplus L^{\vee}, \\
& \widetilde{1}=(1,0,0), \\
& \widetilde{N}(a, w, \check{w})=a^{3}+N_{L}(w)+\check{N}_{L}(\check{w})-3 a\langle w, \check{w}\rangle \\
& (a, w, \check{w})^{\check{\sharp}}=\left(a^{2}-\langle w, \check{w}\rangle, \check{w}^{\sharp}-a w, w^{\sharp}-\check{w} a\right)
\end{aligned}
$$

for $a \in A, w \in L, \check{w} \in L^{\vee}$, then $(\widetilde{N}, \widetilde{\sharp}, \widetilde{1})$ is a cubic form with adjoint and base point on $\widetilde{\mathcal{J}}$ and has trace form

$$
\widetilde{T}((a, w, \check{w}),(c, v, \check{v}))=3 a c+3\langle w, \check{v}\rangle+3\langle v, \check{w}\rangle .
$$

The Jordan algebra $A=\mathcal{J}(\widetilde{N}, \widetilde{\sharp}, \widetilde{1})$ over $R$ is commutative and associative and the nondegenerate cubic form $\widetilde{N}$ permits composition. $A(P)$ is a cubic étale algebra over $k(P)$ for all $P \in \operatorname{Spec} R$. 
Lemma 5. Let $N: A \rightarrow R$ be a nondegenerate cubic form on $A$ permitting composition.

(i) Suppose $A$ can be written as the direct sum of two non-zero ideals $A_{1}, A_{2}$ of $A$. Then $A=R \oplus A_{2}$ where $A_{2}$ is a composition algebra over $R$ with norm $n$ and $N\left(x_{1}+x_{2}\right)=x_{1} n\left(x_{2}\right)$ is absolutely indecomposable.

(ii) Suppose $A$ can be written as the direct sum of three non-zero ideals $A_{1}, A_{2}, A_{3}$ of $A$. Then $A=R \oplus R \oplus R$ and $N\left(x_{1}+x_{2}+x_{3}\right)=x_{1} x_{2} x_{3}$.

Proof. (1) Suppose $A$ can be written as the direct sum of two non-zero ideals. Then $A=A_{1} \oplus A_{2}$ and $N\left(x_{1}+x_{2}\right)=n_{1}\left(x_{1}\right) n\left(x_{2}\right)$ with $n_{1}\left(x_{1}\right)=x_{1}$ and $n$ a nondegenerate quadratic form permitting composition. Therefore $A=R \oplus A_{2}$ where $A_{2}$ is an algebra over $R$ with a nondegenerate quadratic form $n$ permitting composition and $N\left(x_{1}+x_{2}\right)=x_{1} n\left(x_{2}\right)$ is absolutely indecomposable. Since $R$ is a domain, $A_{2}$ has full support, hence it is a composition algebra over $R$.

(2) Suppose $A$ can be written as the direct sum of three non-zero ideals $A=A_{1} \oplus A_{2} \oplus$ $A_{3}$. Then $N\left(x_{1}+x_{2}+x_{3}\right)=n_{1}\left(x_{1}\right) n_{2}\left(x_{2}\right) n_{3}\left(x_{3}\right)$ with $n_{i}$ linear forms permitting composition, $i=1,2,3$. Hence $A=R \oplus R \oplus R$ and $N\left(x_{1}+x_{2}+x_{3}\right)=x_{1} x_{2} x_{3}$.

From now on suppose that $k(P)$ is an infinite field for all $P \in \operatorname{Spec} R$.

Proposition 3. Let $N: A \rightarrow R$ be a quartic form on A permitting composition. Then one of the following holds:

(i) $A=R$ and $N(x)=x^{4}$.

(ii) $A$ is a commutative associative separable $R$-algebra of rank 2 or 3. If $A$ has rank 3, then $A(P)$ cannnot be a cubic field extension of $k(P)$ for all $P \in \operatorname{Spec} R$.

(iii) $A$ has rank 4 and is an associative separable $R$-algebra. If, in particular, $A$ is not commutative, then $A\left(P_{0}\right)$ is a quaternion algebra over $k\left(P_{0}\right)$ for at least one $P_{0}$.

(iv) $A$ has rank 5 or 6 and $A$ is an associative separable $R$-algebra, but not commutative.

(v) $A$ has rank 8 and $A$ is not commutative. If $A$ is not associative, then $A\left(P_{0}\right)$ is an octonion algebra over $k\left(P_{0}\right)$ for some $P_{0} \in \operatorname{Spec} R$.

(vi) A has rank 9,10 or 12, is not associative, not commutative.

(vii) $A$ has rank 16 and $A$ is not associative. Then $A\left(P_{0}\right)$ is an octonion algebra over some quadratic field extension of $k\left(P_{0}\right)$, for some $P_{0} \in \operatorname{Spec} R$.

(viii) $A$ has rank 16 and $A$ is an Azumaya algebra over $R$ with norm $N$.

In cases (i) to (vii), $N$ is absolutely indecomposable.

Proof. Let $m$ be the rank of $A$, then $m \in\{1,2,3,4,5,6,8,9,10,12,16\}$.

(1) If $m=1$, then $A=R$ and $N(x)=x^{4}$.

(2) If $m=2$, then $A(P)$ is isomorphic to a quadratic étale algebra over $k(P)$ and $N(P)=\left(x_{1}+x_{2}\right)=x_{1}^{2} x_{2}^{2}$ is absolutely indecomposable for all $P \in \operatorname{Spec} R$, implying that $N$ must be absolutely indecomposable. By the same argument as used in the proof of Proposition $2(2), A$ is a commutative associative $R$-algebra together.

(3) If $m=3$, then $A(P) \cong k(P) \oplus$ "some quadratic étale algebra over $k(P)$ " by [S3, Lemma 2] and $N(P)$ is absolutely indecomposable for all $P \in \operatorname{Spec} R$. Thus $A$ is commutative, associative and separable and $N$ absolutely indecomposable. 
(4) If $m=4$, then $A(P)$ is either a quaternion algebra over $k(P)$, a separable quartic field extension over $k(P)$, a quadratic étale algebra over some quadratic field extension of $k(P)$ or the direct sum of two quadratic étale algebras over $k(P)$. Hence $A$ is associative and separable. In particular, if $A$ is not commutative, then $A\left(P_{0}\right)$ must be a quaternion algebra over $R$ for a $P_{0} \in \operatorname{Spec} R$.

(5) If $m=5$, then $A(P) \cong k(P) \oplus$ "some quaternion algebra over $k(P)$ " for all $P \in$ Spec $R$ and $N(P)$ is absolutely indecomposable. Thus $A$ is associative and separable, but not commutative, and $N$ absolutely indecomposable.

(6) If $m=6$ then $A(P)$ is isomorphic to the direct sum of a quadratic étale algebra and some quaternion algebra for all $P \in \operatorname{Spec} R$. Thus $A$ is associative and separable, not commutative, and $N$ absolutely indecomposable.

(7) If $m=8$ then $A(P)$ is either an octonion algebra over $k(P)$, a quaternion algebra over some quadratic field extension of $k(P)$, or the direct sum of two quaternion algebras over $k(P)$. Hence $A$ is not commutative and $N$ absolutely indecomposable. If, in particular, $A$ is not associative, then $A\left(P_{0}\right)$ is an octonion algebra over $k\left(P_{0}\right)$ for some $P_{0} \in \operatorname{Spec} R$ and $N\left(P_{0}\right)$ is the square of its norm.

(8) If $m=9$, then $A(P) \cong k(P) \oplus$ "some octonion algebra over $k(P)$ " for all $P \in$ Spec $R$. Thus $A$ is not associative, not commutative, and $N$ absolutely indecomposable.

(9) If $m=10$, then $A(P)$ is isomorphic to the direct sum of a quadratic étale algebra and some octonion algebra over $k(P)$, or $A(P) \cong k(P) \oplus$ "some Azumaya algebra of degree 3 over $k(P)$ ". Thus $A$ is not associative, not commutative and $N$ absolutely indecomposable.

(10) If $m=12$, then $A(P)$ is isomorphic to the direct sum of some octonion algebra and some quaternion algebra for all $P \in \operatorname{Spec} R$. Again $A$ is not commutative, not associative and $N$ absolutely indecomposable.

(11) If $m=16$ and $A$ is associative, then $A(P)$ is an central simple algebra over $k(P)$ for all $P \in \operatorname{Spec} R$. Hence $A$ is an Azumaya algebra over $R$ with norm $N$. If $A$ is not associative, then $A\left(P_{0}\right)$ is an octonion algebra over some quadratic field extension of $k\left(P_{0}\right)$, for some $P_{0} \in \operatorname{Spec} R$.

Lemma 6. Let $N: A \rightarrow R$ be a nondegenerate quartic form $N$ on A permitting composition.

(1) Suppose $A$ can be written as the direct sum of two non-zero ideals. Then $A=A_{1} \oplus A_{2}$ and $N\left(x_{1}+x_{2}\right)=n_{1}\left(x_{1}\right) n_{2}\left(x_{2}\right)$. Either $A_{1}$ and $A_{2}$ are composition algebras and $n_{1}$ and $n_{2}$ are their norms, or $A_{1}=R, n_{1}\left(x_{1}\right)=x_{1}$ and $n_{2}$ is a nondegenerate cubic form permitting composition.

(2) Suppose $A$ can be written as the direct sum of three non-zero ideals. Then $A \cong$ $R \oplus R \oplus A_{3}$ and $N\left(x_{1}+x_{2}+x_{3}\right)=x_{1} x_{2} n_{3}\left(x_{3}\right)$ with $A_{3}$ a composition algebra over $R$ with norm $n_{3}$.

(3) Suppose $A$ can be written as the direct sum of four non-zero ideals. Then $A \cong$ $R \oplus R \oplus R \oplus R$ and $N\left(x_{1}+x_{2}+x_{3}+x_{4}\right)=x_{1} x_{2} x_{3} x_{4}$.

Each $N$ is absolutely indecomposable. 
Proof. (1) Suppose $A$ can be written as the direct sum of two non-zero ideals. Then $A=A_{1} \oplus A_{2}$ and $N\left(x_{1}+x_{2}\right)=n_{1}\left(x_{1}\right) n_{2}\left(x_{2}\right)$ with $n_{1}$ and $n_{2}$ being either two nondegenerate quadratic forms permitting composition, or $A_{1}=R, n_{1}\left(x_{1}\right)=x_{1}$ and $n_{2}$ is a nondegenerate cubic form permitting composition (Theorem 4 (i)) Since $R$ is a domain, $A_{1}$ and $A_{2}$ have full support in both cases.

(2) Suppose $A$ can be written as the direct sum of three non-zero ideals. Then $A \cong$ $A_{1} \oplus A_{2} \oplus A_{3}$ with $A_{1}=A_{2}=R$, and $N\left(x_{1}+x_{2}+x_{3}\right)=n_{1}\left(x_{1}\right) n_{2}\left(x_{2}\right) n_{3}\left(x_{3}\right), n_{1}\left(x_{1}\right)=$ $x_{1}, n_{2}\left(x_{2}\right)=x_{2}$ and $n_{3}$ a nondegenerate quadratic form permitting composition (Theorem 4 (i)). Thus $A_{3}$ is a composition algebra over $R$, since $R$ is a domain.

The rest of the assertion is clear.

\section{EXAMPLES}

Let $k$ be an infinite field. Let $A$ be a unital nonassociative algebra of constant rank over $R$ which is finitely generated projective and faithful as $R$-module. Let $\operatorname{Cay}(D, \mu)$ be the classical Cayley-Dickson doubling of the composition algebra $D$ with scalar $\mu \in R^{\times}$(cf. for instance $[\mathrm{P}])$.

5.1. Let $R=k[t]$ be the polynomial ring over $k$. Suppose that $k$ has characteristic 0 or greater than 3 and that there exists a nondegenerate cubic form on $A$ permitting composition. If $A$ is the direct sum of two non-zero ideals, then $A=R \oplus C$ and $N\left(x_{1}+x_{2}\right)=$ $x_{1} n_{C}\left(x_{2}\right)$, where $C=C_{0} \otimes R$ is a composition algebra defined over $k$ with norm $n_{C}[\mathrm{P}, 6.8]$. Hence both $A=R \oplus C$ and $N$ are defined over $k$ and $N$ is absolutely indecomposable.

If $n_{l / k}$ is the norm of a cubic field extension of $k$, then $N=n_{l / k} \otimes_{k} R$ is another example of an indecomposable cubic form over $R$ permitting composition, again $N$ is defined over $k$.

Suppose now that $k$ has characteristic 0 or greater than 4 and that there exists a nondegenerate quartic form on $A$ permitting composition. The following are examples of such an $(A, N)$ :

(i) $A=R$ and $N(x)=x^{4}$;

(ii) A composition algebra $A$ over $R$ with norm $n$ and $N(x)=n(x)^{2}$. Then both $A$ and $N$ are defined over $k$ and $N$ is absolutely indecomposable.

(iii) A separable quartic ring extension $A$ of $R$ and $N$ its norm, e.g. $N=n_{l / k} \otimes_{k} R$ where $n_{l / k}$ is the norm of a separable quartic field extension of $k$.

(iii) A composition algebra $A$ of constant rank 4 or 8 over its center, which is a separable quadratic ring extension $R^{\prime}$ of $R$. $A$ has a (unique) norm $n_{A / R^{\prime}}$ and $N(x)=n_{R^{\prime} / R}\left(n_{A / R^{\prime}}(x)\right)$. Thus $R^{\prime}$ is isomorphic to $R \oplus R$ or to $k(\sqrt{c}) \otimes_{k} R$. Say $R^{\prime}=k(\sqrt{c}) \otimes_{k} R \cong k(\sqrt{c})[t]$. Then either $A$ is isomorphic to $\operatorname{Mat}_{2}\left(R^{\prime}\right)$ or Zor $R^{\prime}$, or it is without zero divisors and defined over $k(\sqrt{c})$.

(iv) An Azumaya algebra $A$ of rank 16 over $R$ and $N$ its norm.

(v) $A=C \oplus D$ and $N\left(x_{1}+x_{2}\right)=n_{C}\left(x_{1}\right) n_{D}\left(x_{2}\right)$ with $C, D$ two composition algebras over $R$ which are defined over $k$. $A$ and $N$ are defined over $k$ with norms $n_{C}, n_{D} . N$ is absolutely indecomposable.

(vi) $A=R \oplus A_{2}$, where $A_{2}$ is an algebra over $R$ with a cubic form $n_{2}$ permitting composition; that means, $N$ is absolutely indecomposable. For instance, 
(1) $A=R \oplus R$ and $N\left(x_{1}+x_{2}\right)=x_{1} x_{2}^{3}$;

(2) $A=R \oplus R \oplus A_{2}$ and $N\left(x_{1}+x_{2}+x_{3}\right)=x_{1} x_{2} n\left(x_{3}\right)$, where $A_{2}$ is a cubic étale or an Azumaya algebra of rank 9 over $R$ and $n$ its norm;

(3) $A=R \oplus R \oplus C$ and $N\left(x_{1}+x_{2}+x_{3}\right)=x_{1} x_{2} n_{C}\left(x_{3}\right)$, where $C$ is a composition algebra over $R$ defined over $k$ with norm $n_{C}$. $A$ and $N$ are defined over $k$.

(vii) $A=R \oplus R \oplus C$ and $N\left(x_{1}+x_{2}+x_{3}\right)=x_{1} x_{x} n_{C}\left(x_{3}\right)$, where $C$ is a composition algebra over $R$ Both $A$ and $N$ are defined over $k, N$ is absolutely indecomposable.

5.2. Let $R=k\left[t, \frac{1}{t}\right]$ be the ring of Laurent polynomials over $k$. Suppose that $k$ has characteristic 0 or greater than 3 and that there exists a nondegenerate cubic form on $A$ permitting composition. If $A$ is the direct sum of two non-zero ideals, then $A=R \oplus C$ where $C$ is a composition algebra over $R$ with norm $n_{C}$ and $N\left(x_{1}+x_{2}\right)=x_{1} n_{C}\left(x_{2}\right)$. Thus $N$ is absolutely indecomposable and either $C$ (hence $A$ and $N$ ) is defined over $k$ or it is isomorphic to $\operatorname{Cay}(D, \mu t)$, where $D$ is a composition algebra without zero divisors of half the rank of $C$ which is defined over $k$, and $\mu \in k^{\times}$arbitrary [Pu1].

Other examples of absolutely indecomposable cubic forms over $R$ permitting composition are of the type $N=n_{l / k} \otimes_{k} R$ where $n_{l / k}$ is the norm of a cubic field extension of $k$, or of the kind $A=J(R, \mu t)$ and $N(u, v, w)=u^{3}+\mu t v^{3}+\mu^{-1} t^{-1} w^{3}-3 \mu t u v w$ where $J(R, \mu t)$ is the first Tits construction starting with $R, \mu \in k^{\times}$; i.e., $J(R, \mu t)=R(\sqrt[3]{\mu t})$, cf. Example 1 .

Suppose now that $k$ has characteristic 0 or greater than 4 and that there exists a nondegenerate quartic form on $A$ permitting composition. The following are examples of such $(A, N)$ :

(i) $A=R$ and $N(x)=x^{4}$.

(ii) $A$ a composition algebra over $R$ with norm $n$ and $N(x)=n(x)^{2}$. Then $A$ is split and isomorphic to $R \oplus R, \operatorname{Mat}_{2}(R)$ or Zor $R$, or $A=A_{0} \otimes_{k} R$ with $A_{0}$ a composition division algebra over $k$. Consider the non-split case: If it has rank 2 it is either isomorphic to $k(\sqrt{c}) \otimes_{k} R$ for some $c \in k^{\times}$which is not a square, or to $\operatorname{Cay}(R, \mu t)$ with $\mu \in k^{\times}$. Every composition algebra of rank greater than 2 without zero divisors is either defined over $k$ or it is isomorphic to $\operatorname{Cay}(T, \mu t)$ where $T$ is a composition algebra without zero divisors of half the rank which is defined over $k$, and $\mu \in k^{\times}$arbitrary.

(iii) A separable quartic ring extension of $R$ with norm $N$.

(iv) A composition algebra of constant rank 4 or 8 with center $R^{\prime}$, which is a separable quadratic ring extension of $R$ with (unique) norm $n_{A / R^{\prime}}$ and $N(x)=n_{R^{\prime} / R}\left(n_{A / R^{\prime}}(x)\right.$ ).

In particular, it is possible that $R^{\prime}$ is isomorphic to $k(\sqrt{c}) \otimes_{k} R$ or to $\operatorname{Cay}(R, \mu t)$ with $\mu \in k^{\times}$(unless $R^{\prime}$ is not étale, in that case there might be others). Suppose that $R^{\prime}=$ $k(\sqrt{c}) \otimes_{k} R \cong k(\sqrt{c})\left[t, \frac{1}{t}\right]$ for some quadratic field extension $k(\sqrt{c})$ of $k$. Then $A$ is isomorphic to $\operatorname{Mat}_{2}\left(R^{\prime}\right)$ or Zor $R^{\prime}$, or it is a composition division algebra over $R^{\prime}$. It is either defined over $k(\sqrt{c})$ or it is isomorphic to Cay $(T, \mu t)$ where $T$ is a composition algebra without zero divisors of half the rank which is defined over $k(\sqrt{c})$, and $\mu \in k(\sqrt{c})^{\times}$arbitrary.

(iv) An Azumaya algebra of rank 16 over $R$ with norm $N$.

(v) $A=C \oplus D$ and $N\left(x_{1}+x_{2}\right)=n_{C}\left(x_{1}\right) n_{D}\left(x_{2}\right)$ with $C, D$ two composition algebras over $R$ which are defined over $k$. $N$ is absolutely indecomposable. 
(vi) $A=R \oplus A_{2}$, where $A_{2}$ is an algebra over $R$ with a cubic form $n_{2}$ permitting composition; so $N$ is absolutely indecomposable.

(vii) $A=R \oplus R \oplus C$ and $N\left(x_{1}+x_{2}+x_{3}\right)=x_{1} x_{x} n_{C}\left(x_{3}\right)$, where $C$ is a composition algebra over $R$ with norm $n_{C}$; i.e., $C$ is defined over $k$ or isomorphic to Cay $(T, \mu t)$ where $T$ is a composition algebra without zero divisors of half the rank which is defined over $k$, $\mu \in k^{\times}$arbitrary. $N$ is absolutely indecomposable.

\section{Forms PERMitTING COMPOSITION OVER LOCALLY RINGED SPACES}

Let $X$ be a locally ringed space with structure sheaf $\mathcal{O}_{X}$ such that $d ! \in H^{0}\left(X, \mathcal{O}_{X}^{\times}\right)$.

An $\mathcal{O}_{X}$-algebra $\mathcal{A}$ is called alternative if $x^{2} y=x(x y)$ and $y x^{2}=(y x) x$ for all sections $x, y$ of $\mathcal{A}$ over the same open subset of $X$. An associative $\mathcal{O}_{X}$-algebra $\mathcal{A}$ is called an Azumaya algebra if $\mathcal{A}_{P} \otimes_{\mathcal{O}_{P, X}} k(P)$ is a central simple algebra over $k(P)$ for all $P \in X$.

Let $\mathcal{A}$ be an $\mathcal{O}_{X}$-algebra together with a nondegenerate form $N: \mathcal{A} \rightarrow \mathcal{O}_{X}$ of degree $d$ permitting composition; i.e., $N(x y)=N(x) N(y)$ for all sections $x, y$ of $\mathcal{A}$ over the same open subset of $X$. Let $1=1_{\mathcal{A}} \in H^{0}(X, \mathcal{A})$ be the unit element of $\mathcal{A}$. Suppose always that $N(1)=1$. Then $\mathcal{A}$ has full support.

Remark 7. (i) Let $\mathcal{A}=\mathcal{A}_{1} \oplus \mathcal{A}_{2}$ be the direct sum of two non-zero ideals of $\mathcal{A}$. Since $\mathcal{A}$ is locally free of finite rank as $\mathcal{O}_{X}$-module by our convention 2.4, so are $\mathcal{A}_{1}$ and $\mathcal{A}_{2}$.

(ii) Let $X$ be a scheme over the affine scheme $Y=\operatorname{Spec} R, H^{0}\left(X, \mathcal{O}_{X}\right)=R$. If $\mathcal{A}$ is globally free as an $\mathcal{O}_{X}$-module then $(\mathcal{A}, N)$ is defined over $R$. (The proof is analogous to the one of $[\mathrm{P}, 1.10]$.

Let $\theta: \mathcal{A} \times \cdots \times \mathcal{A} \rightarrow \mathcal{O}_{X}$ be the $d$-linear form associated with $N$. For $i=1, \ldots, d$ define a form $T_{i}: \mathcal{A} \rightarrow \mathcal{O}_{X}$ of degree $i$ via

$$
T_{i}(x)=\left(\begin{array}{c}
n \\
i
\end{array}\right) \theta(x, \ldots, x, 1, \ldots, 1) \quad(i \text {-times } x) .
$$

Then

$$
N(x)=T_{d}(x) \text { and } T_{1}(x)=d \theta(x, 1, \ldots, 1)
$$

for all sections $x$ of $\mathcal{A}$ over the same open subset of $X$.

The form $T: \mathcal{A} \rightarrow \mathcal{O}_{X}, T(x)=T_{1}(x)$ is called the trace. Put $T_{0}(x)=1$ and $T_{d+q}(x)=0$ for $q>0$, then $T(a 1)=\operatorname{da\theta }(1, \ldots, 1)$ for all $a$ in $\mathcal{O}_{X}$. Define $\mathcal{A}_{0}=\operatorname{ker} T$. $\mathcal{A}$ is the direct sum of $\mathcal{O}_{X}$-modules $\mathcal{A}=\mathcal{O}_{X} 1_{\mathcal{A}} \oplus \mathcal{A}_{0}$.

We assume from now on that $d \geq 3$. Our results from Section 3 easily adapt to the setting of locally ringed spaces: $\mathcal{A}$ is alternative and $B: \mathcal{A} \times \mathcal{A} \rightarrow \mathcal{O}_{X}$, with $B(x, y)=T(x y)$ for all sections $x, y$ of $\mathcal{A}$ over the same open subset of $X$, is a nondegenerate symmetric bilinear form on $\mathcal{A}$ which is associative. Every section $x$ of $\mathcal{A}$ over the same open subset of $X$ satisfies

$$
x^{d}-T_{1}(x) x^{d-1}+T_{2}(x) x^{d-2}-\cdots+(-1)^{d} T_{d}(x) 1=0 .
$$

From now on let $X$ be an integral scheme. If $\mathcal{A}=\mathcal{A}_{1} \oplus \cdots \oplus \mathcal{A}_{r}$ with $\mathcal{A}_{i}$ non-zero ideals of $\mathcal{A}$, then

$$
N(x)=N_{1}\left(x_{1}\right) \cdots N_{2}\left(x_{r}\right)
$$


where $x=x_{1}+\cdots+x_{r}, x_{i} \in \mathcal{A}_{i}$ for all $i$, and each $N_{i}$ is a nondegenerate form of degree $d_{i}$ on $\mathcal{A}_{i}, d=d_{1}+\cdots+d_{r}$, which permits composition.

Lemma 7. (i) $\mathcal{A}(P)$ is separable for all $P \in X$.

(ii) If $\mathcal{A}$ is not associative, but $\mathcal{A}(P)$ is simple for all $P \in X$, then there is at least one $P \in X$ such that $\mathcal{A}(P)$ is an octonion algebra over some separable field extension of $k(P)$.

Proof. (i) We know that for all $P \in X, \mathcal{A}(P)$ is a non-zero $k(P)$-algebra such that there exists a nondegenerate form $N(P)$ of degree $d$ on $\mathcal{A}(P)$ permitting composition (2.3 and Lemma 1). Thus $\mathcal{A}(P)$ is a finite dimensional separable $k(P)$-algebra for all $P \in X$.

(ii) follows immediately.

Proposition 4. (i) If $N$ is a cubic form then $\mathcal{A}$ has rank 1, 2, 3, 5 or 9.

(ii) If $N$ is a quartic form, then $\mathcal{A}$ has rank $1,2,3,4,5,6,8,9,10,12$ or 16 .

Proof. For all $P \in \operatorname{Spec} R$ the residue class algebra $\mathcal{A}(p)=\mathcal{A}_{P} \otimes k(P)$ is a nonzero alternative algebra over the residue class field $k(P)$ together with a nondegenerate form $N(P): \mathcal{A}(P) \rightarrow$ $k(P)$ which permits composition (2.3 and Lemma 1$)$. This implies that if $N$ is a cubic form then $\mathcal{A}(P)$ must have rank $1,2,3,5$ or 9 , and if it is a quartic form then $\mathcal{A}(P)$ must have $\operatorname{rank} 1,2,3,4,5,6,8,9,10,12$ or 16 [S1, p. 140].

We now turn to cubic forms permitting composition.

Lemma 8. A nondegenerate cubic form $N$ permitting composition cannot be defined

(i) on a composition algebra over $X$ of constant rank greater than 2;

(ii) on a non-split quadratic étale algebra $\mathcal{A}$ over $X$, unless $\mathcal{A}(P)$ is split for all $P \in X$.

This can be proved by assuming such a form exists and using Lemma 3 locally to obtain a contradiction.

Proposition 5. Suppose that there exists a nondegenerate cubic form $N$ on $\mathcal{A}$ permitting composition. Then $(\mathcal{A}, N)$ satisfies one of the following:

(i) $\mathcal{A}=\mathcal{O}_{X}$ and $N(x)=x^{3}$;

(ii) $\mathcal{A}$ is commutative and associative of rank 2 or 3 , and $N$ is absolutely indecomposable; if $\mathcal{A}$ is a non-split quadratic étale algebra over $X$, then $\mathcal{A}(P)$ must be split for all $P \in X$; if $\mathcal{A}$ has rank 3 then $\mathcal{A}(P)$ is a cubic étale algebra over $k(P)$ for all $P \in X$.

(iii) $\mathcal{A}$ is an associative and not commutative $\mathcal{O}_{X}$-algebra of rank 5 , and $N$ is absolutely indecomposable.

(iv) $\mathcal{A}$ is an Azumaya algebra over $X$ of rank 9 , and $N$ is its reduced norm.

(v) $\mathcal{A}$ has rank 9 and is neither associative nor commutative, $N$ is absolutely indecomposable.

This follows from Proposition 2.

Example 2. There is the following first Tits construction starting with the structure sheaf of an integral scheme $X$ [Ach, 2.25]: Let $\mathcal{L} \in{ }_{3} \operatorname{Pic} X$ and $N: \mathcal{L} \rightarrow \mathcal{O}_{X}$ a nondegenerate cubic form, let $\check{\mathcal{L}}=\operatorname{Hom}_{\mathcal{O}_{X}}\left(\mathcal{L}, \mathcal{O}_{X}\right)$ be its dual and $\langle w, \breve{w}\rangle=\check{w}(w)$ the canonical pairing $\mathcal{L} \times \check{\mathcal{L}} \rightarrow \mathcal{O}_{X}$. There exists a uniquely determined cubic norm $\check{N}: \check{\mathcal{L}} \rightarrow \mathcal{O}_{X}$ and uniquely 
determined adjoints $\sharp: \mathcal{L} \rightarrow \check{\mathcal{L}}$ and $\check{\sharp}: \check{\mathcal{L}} \rightarrow \mathcal{L}$ such that the identities listed in Example 1 (with $N$ instead of $N_{L}$ ) hold for $w, w^{\prime}$ in $\mathcal{L}, \check{w}$ in $\check{\mathcal{L}}$ [Ach, 2.13]. Define

$$
\begin{aligned}
& \tilde{\mathcal{J}}=\mathcal{O}_{X} \oplus \mathcal{L} \oplus \check{\mathcal{L}}, \\
& \widetilde{1}=(1,0,0) \in H^{0}(X, \mathcal{J}), \\
& \widetilde{N}(a, w, \check{w})=a^{3}+N(w)+\check{N}(\check{w})-3 a\langle w, \check{w}\rangle \\
& (a, w, \check{w})^{\sharp}=\left(a^{2}-\langle w, \check{w}\rangle, \check{w}^{\sharp}-a w, w^{\sharp}-\check{w} a\right)
\end{aligned}
$$

for $a \in \mathcal{O}_{X}, w \in \mathcal{L}, \check{w} \in \check{\mathcal{L}}$, then $(\widetilde{N}, \widetilde{\sharp}, \widetilde{1})$ is a cubic form with adjoint and base point on $\widetilde{\mathcal{J}}$ and has trace form

$$
\widetilde{T}((a, w, \check{w}),(c, v, \check{v}))=3 a c+3\langle w, \check{v}\rangle+3\langle v, \check{w}\rangle .
$$

The induced (commutative associative) Jordan algebra $J(\widetilde{N}, \widetilde{\sharp}, \widetilde{1})$ is denoted by $J(\mathcal{A}, \mathcal{P}, N)$. This construction yields examples of commutative associative algebras $\mathcal{A}=\mathcal{J}\left(\mathcal{O}_{X}, \mathcal{L}, N\right)$ of constant rank 3 which admit a nondegenerate cubic form $\widetilde{N}$ permitting composition. $\mathcal{A}(P)$ is a cubic étale algebra over $k(P)$ for all $P \in \operatorname{Spec} X$.

Example 3 (Pu2). Let $k$ be a field of characteristic 0 . Let $X$ be an elliptic curve $X$ over $k$. Let $\mathcal{N}_{i}$ denote a line bundle of order 3 on $X$ with $\mathcal{N}_{0}=\mathcal{O}_{X}$. We have ${ }_{3} \operatorname{Pic}(X)=$ $\left\{\mathcal{N}_{i} \mid 0 \leq 1 \leq m\right\}$ for some even integer $0 \leq m \leq 8$. Every first Tits construction over $X$ starting with $\mathcal{O}_{X}$ is isomorphic to $\mathcal{A}=\mathcal{J}\left(\mathcal{O}_{X}, \mathcal{N}_{i}, N_{i}\right)$ where $N_{i}$ is a nondegenerate cubic form on $\mathcal{N}_{i}$. By the Theorem of Krull-Schmidt, if $\mathcal{N}_{i} \not \mathcal{N}_{j}$ and $\mathcal{N}_{i} \not \mathcal{N}_{j}^{\vee}$ then $\mathcal{J}\left(\mathcal{O}_{X}, \mathcal{N}_{i}, N_{i}\right) \neq \mathcal{J}\left(\mathcal{O}_{X}, \mathcal{N}_{j}, N_{j}\right) . \mathcal{J}\left(\mathcal{O}_{X}, \mathcal{N}_{0}, N_{0}\right)$ is defined over $k$.

\section{Curves of genus zero}

Lemma 9. Let $X$ be a curve of genus zero over a field $k$ of characteristic not 2 or 3. Let $\mathcal{A}$ be an algebra over $X$ of rank 2 carrying a nondegenerate form $N$ permitting composition. If $N$ is a cubic or quartic form then $(\mathcal{A}, N)$ is defined over $k$.

Proof. $\mathcal{A}$, together with the nondegenerate symmetric bilinear form $T_{\mathcal{A}}$, is a nondegenerate bilinear space over $X$. If $X$ is rational, $[\mathrm{P}, 5.4]$ shows that $\mathcal{A}$ decomposes into the orthogonal sum of $\mathcal{O}_{X}$-modules of the kind $\mathcal{O}_{X}\left(m_{i}\right) \oplus \mathcal{O}_{X}\left(-m_{i}\right)$ for suitable $m_{i}>0$. Hence, if $\mathcal{A}$ has rank two, it must be globally free as an $\mathcal{O}_{X}$-module.

If $X$ is nonrational, there is a field extension $k^{\prime} / k$ such that $X^{\prime}=X \times_{k} k^{\prime}$ becomes rational. If $\mathcal{A}$ is an algebra as in our assumption then so is $\mathcal{A} \otimes \mathcal{O}_{X^{\prime}}$ and since this is globally free, so is $\mathcal{A}$. By Remark 7 (ii), in both cases $(A, N)$ is defined over $k$.

Lemma 10. Let $X$ be a curve of genus zero over a field $k$ of characteristic not 2 or 3. Every first Tits construction over $X$ starting with $\mathcal{O}_{X}$ is defined over $k$.

Proof. Every first Tits construction over $X$ starting with $\mathcal{O}_{X}$ is of the kind $J\left(\mathcal{O}_{X}, \mathcal{L}, N_{\mathcal{L}}\right)$, where $\mathcal{L} \in{ }_{3} \operatorname{Pic} X$ (Example 2). However, $\operatorname{Pic} X \cong \mathbb{Z}$, so we only have $J\left(\mathcal{O}_{X}, \mathcal{O}_{X}, \mu\right) \cong$ $J(k, \mu) \otimes_{k} \mathcal{O}_{X}, \mu \in k^{\times}$. 
7.1. Let $k$ be a field of characteristic 0 and $X$ be a curve of genus zero over $k$. If $X$ is not rational, let $D_{0}=(a, b)_{k}$ be the quaternion division algebra associated with $X$. Let $\mathcal{A}$ be an algebra (automatically of constant rank) over $X$ such that there exists a nondegenerate cubic form $N$ on $\mathcal{A}$ permitting composition.

(1) If $\mathcal{A}$ has rank 1, then $\mathcal{A}=\mathcal{O}_{X}$ and $N(x)=x^{3}$.

(2) If $\mathcal{A}$ has rank 3 it is commutative associative and $N$ is absolutely indecomposable. For instance, let $k^{\prime}$ be a cubic field extension of $k$, then $\mathcal{A}=k^{\prime} \otimes_{k} \mathcal{O}_{X}$ carries a nondegenerate cubic form permitting composition.

(3) If $\mathcal{A}$ is the direct sum of two non-zero ideals $\mathcal{A}=\mathcal{A}_{1} \oplus \mathcal{A}_{2}$, then $N\left(x_{1}+x_{2}\right)=$ $N_{1}\left(x_{1}\right) N_{2}\left(x_{2}\right)$ and $N_{1}$ must be a nondegenerate linear form and $N_{2}$ a nondegenerate quadratic one (or vice versa). It follows that $\mathcal{A}_{1}=\mathcal{O}_{X}, N_{1}=i d$, and $\mathcal{A}_{2}$ is an algebra of degree 2 over $X$ of constant rank with a nondegenerate quadratic form $N_{2}$ permitting composition. Therefore $\mathcal{A}_{2}$ is a composition algebra over $X$ of constant rank with norm $N_{2}$. Hence

$$
\mathcal{A}=\mathcal{O}_{X} \oplus \mathcal{C}
$$

with $C$ a quadratic étale algebra, a quaternion or an octonion algebra over $X$, and $N$ is absolutely indecomposable. (Note that $\mathcal{A}$ cannot be the direct sum of more than two non-zero ideals.) By [P, 4.4], one of the following holds:

(i) $\mathcal{C}$ (and thus $\mathcal{A}$ ) is defined over $k$.

(ii) $\mathcal{C}$ is a split quaternion or octonion algebra.

(iii) $X$ is not rational and $\mathcal{C} \cong \operatorname{Cay}\left(\mathcal{D}, \mathcal{P}, N_{\mathcal{P}}\right)$, where $\mathcal{D}=D_{0} \otimes \mathcal{O}_{X}, \mathcal{P}$ is a locally free right $\mathcal{D}$-module of rank one and norm one, and $N_{\mathcal{P}}$ is a norm on it. More precisely, let $\mathcal{E}_{0}$ be the indecomposable $\mathcal{O}_{X}$-module of rank 2 described in [P, 4.3]. Then we know that $\mathcal{P}=\mathcal{P}_{1} \oplus \mathcal{P}_{2}$ with $\mathcal{P}_{1}=\mathcal{L}\left(m P_{0}\right) \otimes \check{\mathcal{E}}_{0}$ and $\mathcal{P}_{2}=\mathcal{L}\left((-m+1) P_{0}\right) \otimes \check{\mathcal{E}}_{0}$ for some integer $m \geq 0$ uniquely determined by $\mathcal{C}$, where $P_{0}$ is a closed point of $X$ of minimal degree.

(4) If $\mathcal{A}$ is the direct sum of three non-zero ideals $\mathcal{A}=\mathcal{A}_{1} \oplus \mathcal{A}_{2} \oplus \mathcal{A}_{3}$, then $N\left(x_{1}+x_{2}+\right.$ $\left.x_{3}\right)=N_{1}\left(x_{1}\right) N_{2}\left(x_{2}\right) N_{2}\left(x_{3}\right)$ and $N_{i}$ must be a nondegenerate linear form $(i=1,2,3)$ It follows that $N\left(x_{1}+x_{2}+x_{3}\right)=x_{1} x_{2} x_{3}$ and $\mathcal{A}$ is defined over $k$.

(5) Suppose that $\mathcal{A}$ does not decompose into the direct sum of non-zero ideals.

If $\mathcal{A}$ has rank 9 and is associative then $\mathcal{A}$ is an Azumaya algebra over $X$ of rank 9 and $N$ its reduced norm. If $X$ is rational, then either we have

$$
\mathcal{A} \cong \mathcal{E}_{n d_{X}}\left(\mathcal{O}_{X}\left(m_{1}\right) \oplus \mathcal{O}_{X}\left(m_{2}\right) \oplus \mathcal{O}_{X}\left(m_{3}\right)\right)
$$

with $m_{i} \in \mathbb{Z}$, or both $\mathcal{A}$ and $N$ are defined over $k$ and we have

$$
\mathcal{A} \cong \sigma^{*} D
$$

with $D$ a central simple division algebra over $k$ ([Kn1, VII (3.1.1)], [Kn2], see also [Ach]). If $X$ is nonrational, we can give some examples of Azumaya algebras of rank 9 over $X$ :

For instance, again the trivial case that $\mathcal{A} \cong \sigma^{*} D$ with $D$ a central simple division algebra over $k$. 
For every locally free $\mathcal{O}_{X}$-module $\mathcal{E}$ of constant rank $3, \mathcal{E} n d_{X}(\mathcal{E})$ is an Azumaya algebra of rank 9 where we have the following possibilities for $\mathcal{E}$ :

$$
\mathcal{E}=\mathcal{L}\left(m_{1} P_{0}\right) \otimes \mathcal{E}_{0} \oplus \mathcal{L}\left(m_{2} P_{0}\right) \text { and } \mathcal{E}=\mathcal{L}\left(n_{1} P_{0}\right) \oplus \mathcal{L}\left(n_{2} P_{0}\right) \oplus \mathcal{L}\left(n_{3} P_{0}\right)
$$

with $m_{1}, m_{2}, n_{1}, n_{2}, n_{3} \in \mathbb{Z}$. Hence

(i)

$$
\mathcal{E} n d_{X}(\mathcal{E})=\left[\begin{array}{cc}
\mathcal{E} n d\left(\mathcal{E}_{0}\right) & \mathcal{L}\left(-a P_{0}\right) \otimes \mathcal{E}_{0} \\
\mathcal{L}\left(a P_{0}\right) \otimes \check{\mathcal{E}}_{0} & \mathcal{O}_{X}
\end{array}\right]
$$

$$
\mathcal{E} n d_{X}(\mathcal{E})=\left[\begin{array}{ccc}
\mathcal{O}_{X} & \mathcal{L}\left(c P_{0}\right) & \mathcal{L}\left(b P_{0}\right) \\
\mathcal{L}\left(-c P_{0}\right) & \mathcal{O}_{X} & \mathcal{L}\left((b-c) P_{0}\right) \\
\mathcal{L}\left(-b P_{0}\right) & \mathcal{L}\left((c-b) P_{0}\right) & \mathcal{O}_{X}
\end{array}\right]
$$

with $a=m_{2}-m_{1}, b=n_{1}-n_{3}$ and $c=n_{1}-n_{2}$ [Ach, 4.4].

\section{Degenerate Forms Permitting COMPosition}

8.1. Let us consider forms $N: A \rightarrow R$ permitting composition over rings $R$ with $d ! \in R^{\times}$, where $A$ is a unital nonassociative $R$-algebra which is finitely generated projective as $R$ module. We now look at the case where $N: A \rightarrow R$ is degenerate; i.e., where the radical

$$
\operatorname{rad} N=\left\{x \in A \mid \theta\left(x, x_{2}, \ldots, x_{d}\right)=0 \text { for all } x_{i} \in A\right\}
$$

is non-zero. As before, let $B: A \times A \rightarrow R, B(x, y)=T(x y)$ where $T$ is the trace of $A$.

Lemma 11. Let $A$ be an R-algebra together with a form $N$ of degree $d$ on $A$ permitting composition. Let $D$ be a subalgebra of $A$ which is maximal among all subalgebras $E$ of $A$ which have a nondegenerate restriction $\left.N\right|_{E}$. Then $(A, B)=\left(D,\left.B\right|_{D}\right) \perp\left(D^{\perp},\left.B\right|_{D^{\perp}}\right)$ and $D D^{\perp} \subset D^{\perp}$ as well as $D^{\perp} D \subset D^{\perp}$.

Proof. Since $N_{D}$ is nondegenerate, so is $\left.B\right|_{D}$. Thus $(A, B)=\left(D,\left.B\right|_{D}\right) \perp\left(D^{\perp},\left.B\right|_{D^{\perp}}\right)$ by [Kn1, I(3.6.2)]. Let $x \in D$ and $y \in D^{\perp}$. Then $B(z, x y)=B(z x, y)=0$ and $B(y x, z)=$ $B(y, x z)=0$ for all $z \in D$, thus $x y \in D^{\perp}$ and $y x \in D^{\perp}$. Since $B$ is an associative symmetric bilinear form, $\operatorname{rad} B$ is a two-sided ideal. We have $\operatorname{rad}(B)=\operatorname{rad}(N)$ by Lemma 1 (i).

For $d=2$ and $R$ a field this was proved in [K-S, 1.2].

Remark 8. The radical $\operatorname{rad} N$ of a cubic form $N$ permitting composition on an $R$-algebra $A$ is a two-sided ideal [B-B, Lemma 2] (indeed, this is true for the radical of any form of degree $d$ permitting composition [B]). If $A$ is also an algebra of degree 3 as defined in [B-B], then $\operatorname{rad} N$ is a nilideal. If, additionally, $R$ does not contain any non-zero nilpotent elements, then $\operatorname{rad} N$ is the maximal nilideal of $A$, that means the radical [B-B, Lemma 5].

For quadratic forms permitting composition, the radical can be annihilated by a suitable exponent which depends on the dimension of $A$ ([K-S] or [M2]). For degenerate cubic forms permitting composition of the kind $N(a, x)=a N_{C}(x)$ where $N_{C}$ is a quadratic form permitting composition, the radical can be annihilated by exactly that exponent which depends on the dimension of $C$, since in that case $\operatorname{rad} N_{0}=0 \oplus \operatorname{rad} N_{C}$. 
8.2. Let $X=\mathbb{P}_{R}^{n}$ be the $n$-dimensional projective space over $R$, that is $X=\operatorname{Proj} S$ where $S=R\left[t_{0}, \ldots, t_{n}\right]$ is the polynomial ring in $n+1$ variables over $R$, equipped with the canonical grading $S=\oplus_{m \geq 0} S_{m}$. We have $\operatorname{rank} S_{m}=\left(\begin{array}{c}m+n \\ n\end{array}\right)$. We know that $\mathcal{O}_{X}(m)$ is a locally free $\mathcal{O}_{X}$-module of rank one for each $m \in \mathbb{Z}$ and

$$
\begin{gathered}
H^{0}\left(X, \mathcal{O}_{X}(m)\right)=S_{m} \text { for } m \geq 0, \\
H^{0}\left(X, \mathcal{O}_{X}(m)\right)=0 \text { for } m<0 .
\end{gathered}
$$

Example 4. Let $\mathcal{C}$ be the split octonion algebra

$$
\operatorname{Zor}\left(\mathcal{O}_{X}(l) \oplus \mathcal{O}_{X}(m) \oplus \mathcal{O}_{X}(-l-m), \alpha\right)
$$

over $X$ with norm $n_{C}$ as defined in $[\mathrm{P}, 3.3]$ (l,m positive integers). Let $\mathcal{A}=\mathcal{O}_{X} \oplus \mathcal{C}$ and $N\left(\left(x_{1}, x_{2}\right)\right)=x_{1} n_{C}\left(x_{2}\right)$ for all sections $x_{1}$ in $\mathcal{O}_{X}, x_{2}$ in $\mathcal{C}$. Then $N(1)=1, N$ permits composition, and $N$ is absolutely indecomposable. We get

$$
A=H^{0}(X, \mathcal{A})=R \oplus H^{0}(X, \mathcal{C})=R \oplus\left[\begin{array}{cc}
R & S_{l} \oplus S_{m} \\
S_{l+m} & R
\end{array}\right]
$$

with the algebra multiplication in $H^{0}(X, \mathcal{C})$ as described in $[\mathrm{P}, 3.8] . A$ is an alternative $R$-subalgebra of $S \oplus$ Zor $S$ of rank

$$
3+\left(\begin{array}{c}
l+n \\
n
\end{array}\right)+\left(\begin{array}{c}
m+n \\
n
\end{array}\right)+\left(\begin{array}{c}
(l+m)+n \\
n
\end{array}\right)
$$

and $x^{3}-T_{1}(x) x^{2}+T_{2}(x) x-T_{3}(x) 1=0$ for each $x \in A$. If $n=1$ then $\operatorname{rank}_{R} H^{0}(X, \mathcal{A})=$ $6+2(l+m) \geq 10$ must be even. If $R$ is a field then the cubic form $N_{0}=N(X)$ restricted to the subalgebra

$$
R \oplus\left[\begin{array}{cc}
R & 0 \\
0 & R
\end{array}\right]
$$

of rank 3 is nondegenerate and

$$
\operatorname{rad} N_{0}=0 \oplus \operatorname{rad}\left(H^{0}(X, \mathcal{C})\right)=0 \oplus\left[\begin{array}{cc}
0 & S_{l} \oplus S_{m} \\
S_{l+m} & 0
\end{array}\right]
$$

is the radical of $A[\mathrm{P}, 3.8]$. We have

$$
\left(\operatorname{rad} N_{0}\right)^{2}=0 \oplus\left[\begin{array}{cc}
0 & 0 \\
S_{l+m} & 0
\end{array}\right]
$$

and $\left(\operatorname{rad} N_{0}\right)^{3}=0$.

Example 5. Let $\mathcal{F}=\mathcal{O}_{X}\left(m_{1}\right) \oplus \mathcal{O}_{X}\left(m_{2}\right) \oplus \mathcal{O}_{X}\left(m_{3}\right)$, then $\mathcal{A}=\mathcal{E} n d_{X}(\mathcal{F})$ is an Azumaya algebra over $X$ of constant rank 9 . We have

$$
\mathcal{A}=\left[\begin{array}{ccc}
\mathcal{O}_{X} & \mathcal{O}_{X}(a) & \mathcal{O}_{X}(b) \\
\mathcal{O}_{X}(-a) & \mathcal{O}_{X} & \mathcal{O}_{X}(b-a) \\
\mathcal{O}_{X}(-b) & \mathcal{O}_{X}(a-b) & \mathcal{O}_{X}
\end{array}\right]
$$


with $a=m_{1}-m_{2}, b=m_{1}-m_{3}$, the right hand side being equipped with the usual matrix multiplication. $H^{0}(X, \mathcal{A})$ is a unital associative $R$-algebra of degree 3 which admits a cubic form $N_{0}=N(X): H^{0}(X, \mathcal{A}) \rightarrow H^{0}\left(X, \mathcal{O}_{X}\right)$ permitting composition which satisfies $N_{0}\left(1_{A}\right)=1$.

(1) If $a, b>0$ and $b-a>0$ then

$$
H^{0}(X, \mathcal{A})=\left[\begin{array}{ccc}
R & S_{a} & S_{b} \\
0 & R & S_{b-a} \\
0 & 0 & R
\end{array}\right]
$$

has rank

$$
3+\left(\begin{array}{c}
a+n \\
n
\end{array}\right)+\left(\begin{array}{c}
b+n \\
n
\end{array}\right)+\left(\begin{array}{c}
(b-a)+n \\
n
\end{array}\right)
$$

In particular, if $n=1$ then $\operatorname{rank}_{R} H^{0}(X, \mathcal{A})=6+2 b \geq 8$ must be even. If $R$ is a field then

$$
\operatorname{rad} N_{0}=\left[\begin{array}{ccc}
0 & S_{a} & S_{b} \\
0 & 0 & S_{b-a} \\
0 & 0 & 0
\end{array}\right]
$$

is the radical of $N_{0}$, i.e. the radical of $A$. We get

$$
\left(\operatorname{rad} N_{0}\right)^{2}=\left[\begin{array}{ccc}
0 & 0 & S_{b} \\
0 & 0 & 0 \\
0 & 0 & 0
\end{array}\right]
$$

and $\left(\operatorname{rad} N_{0}\right)^{3}=0$.

(2) If $a=b>0$ then

$$
H^{0}(X, \mathcal{A})=\left[\begin{array}{ccc}
R & S_{a} & S_{a} \\
0 & R & R \\
0 & R & R
\end{array}\right]
$$

has odd rank $5+2\left(\begin{array}{c}a+n \\ n\end{array}\right)$. In particular, if $n=1$ then $\operatorname{rank}_{R} H^{0}(X, \mathcal{A})=5+2(a+1) \geq$ 9. If $R$ is a field then

$$
\operatorname{rad} N_{0}=\left[\begin{array}{ccc}
0 & S_{a} & S_{a} \\
0 & 0 & 0 \\
0 & 0 & 0
\end{array}\right]
$$

and $\left(\operatorname{rad} N_{0}\right)^{2}=0$. Again, $\operatorname{rad} N_{0}$ is the radical of $A$. 
(3) If $a=0$ and $b>0$ then

$$
H^{0}(X, \mathcal{A})=\left[\begin{array}{ccc}
R & R & S_{b} \\
R & R & S_{b} \\
0 & 0 & R
\end{array}\right]
$$

has odd rank $5+2\left(\begin{array}{c}b+n \\ n\end{array}\right)$. In particular, if $n=1$ then $\operatorname{rank}_{R} H^{0}(X, \mathcal{A})=5+2(b+1) \geq$ 9. If $R$ is a field then

$$
\operatorname{rad} N_{0}=\left[\begin{array}{ccc}
0 & 0 & S_{b} \\
0 & 0 & S_{b} \\
0 & 0 & 0
\end{array}\right]
$$

and $\left(\operatorname{rad} N_{0}\right)^{2}=0$.

Analogously, one can construct classes of degenerate forms of degree higher than 3 over $R$ which permit composition.

Acknowledgements: The author would like to acknowledge the support of the "GeorgThieme-Gedächtnisstiftung" (Deutsche Forschungsgemeinschaft) during her stay at the University of Trento, and to thank the Department of Mathematics of the University of Trento for its hospitality.

\section{REFERENCES}

[Ach] Achhammer, G., Albert Algebren über lokal geringten Räumen. PhD Thesis, FernUniversität Hagen, 1995.

[A] Albert, A. A., Quadratic forms permitting composition. Ann. of Math. (2) (1942), 161-177.

[B] Bergmann, A., Formen auf Moduln über kommutativen Ringen beliebiger Charakteristik. J. Reine Angew. Math. 219 (1965), 113-156.

[B-B] Baumgartner, E., Bergmann, A., Nichtausgeartete Kompositionsalgebren vom Grad 3. J. Reine Angew. Math. 268/269 (1974), 324-337.

[H] Hartshorne, R., "Algebraic geometry". Graduate Texts in Mathematics, vol. 52, Springer-Verlag, Berlin-Heidelberg-New York, 1977.

[K] Keet, A., Decomposition of a higher degree form. Comm. Alg. 30 (10) (2002), 4945-4963.

[Kn1] Knus, M.-A., "Quadratic and hermitian forms over rings". Springer-Verlag, Berlin-Heidelberg-New York, 1991.

$[\mathrm{Kn} 2] \quad$ Knus, M.-A., Quaternionic modules over $\mathbb{P}^{2}(\mathbb{R})$. Proceedings of the conference "Brauer groups in ring theory and algebraic geometry", Antwerpen 1981, eds. F. van Oystaeyen and A. Verschoren, Lecture Notes in Math. 917, Springer-Verlag, New York - Heidelberg - Berlin, 1982.

[K-S] Kunze, R. A., Scheinberg, S., Alternative algebras having scalar involutions. Pac. J. Math. 124 (1) (1986), 159-172.

[L-L] Legrand, S., Legrand, D., Algébres sur un anneau munies d'une forme multiplicative de degré $n$. J. Reine Angew. Math. 299/300 (1978), 171-184.

[M1] McCrimmon, K., A proof of Schafer's conjecture for infinite-dimensional forms permitting composition. J. Algebra 5 (1967), 72-83.

[M2] McCrimmon, K., Nonassociative algebras with scalar involution. Pacific J. Math. 116 (1985), 85-108.

[P] Petersson, H.P., Composition algebras over algebraic curves of genus zero. Trans. Amer. Math. Soc. 337(1) (1993), 473-491. 
[P-R] Petersson, H. P., Racine, M. L., Jordan algebras of degree 3 and the Tits process. J. Algebra 98 (1986) (1), 211-243.

[Pr] Prószyński, A., On orthogonal decomposition of homogenous polynomials. Fundamenta Math. 98 (3) (1978), 201-217.

[Pu1] Pumplün, S., Composition algebras over the Laurent polynomials. Comm. Alg. 25(1) (1997), 229233.

[Pu2] Pumplün, S., Albert algebras over curves of genus zero and one. Preprint, 2007.

[R] Roby, N., Lois polynômes et lois formelles en théorie des modules. Ann. Sci. Ecole Norm. Sup. $3^{e}$ ser. t. 80 (1963), 213- 348.

[S1] Schafer, R.D., Forms permitting composition. Adv. Math. 4 (1970), 127-148.

[S2] Schafer, R.D., On cubic forms permitting composition. Proc. Amer. Math. Soc. 10 (1959), 917-925.

[S3] Schafer, R.D., On forms of degree $n$ permitting composition. J. of Mathematics and Mechanics (1963) 12, 777-792.

[S4] Schafer, R.D., "An Introduction to Nonassociative Algebras". Academic Press, Inc., New York, 1966 (reprinted by Dover Publications, Inc., New York, 1995).

[S11] Slater, M., Alternative rings with d.c.c. I. J. Algebra 11 (1969), 102-110.

[S12] Slater, M., The socle of an alternative ring. J. Algebra 14 (1970), 443-463.

E-mail address: susanne.pumpluen@nottingham.ac.uk

School of Mathematics, University of Nottingham, University Park, Nottingham NG7 2RD, United Kingdom 